\title{
Gradhiva
}

GRADHI

Revue d'anthropologie et d'histoire des arts

$17 \mid 2013$

L'esthétique du geste technique

\section{Aurélie Helmlinger. Pan Jumbie. Mémoire sociale et musicale dans les steelbands (Trinidad et Tobago)}

Nanterre, Société d'ethnologie, coll. « Hommes et musiques », 2012

\section{Tommaso Montagnani}

\section{(2) OpenEdition}

\section{Journals}

Édition électronique

URL : http://journals.openedition.org/gradhiva/2685

DOI : $10.4000 /$ gradhiva.2685

ISSN : $1760-849 x$

\section{Éditeur}

Musée du quai Branly Jacques Chirac

Édition imprimée

Date de publication : 16 mai 2013

Pagination : 220-221

ISBN : $978-2-35744-049-74$

ISSN : 0764-8928

\section{Référence électronique}

Tommaso Montagnani, « Aurélie Helmlinger. Pan Jumbie. Mémoire sociale et musicale dans les steelbands (Trinidad et Tobago) », Gradhiva [En ligne], 17 | 2013, mis en ligne le 28 mai 2013, consulté le 22 septembre 2020. URL : http://journals.openedition.org/gradhiva/2685 ; DOI : https://doi.org/ $10.4000 /$ gradhiva.2685

Ce document a été généré automatiquement le 22 septembre 2020

(c) musée du quai Branly 


\section{Aurélie Helmlinger. Pan Jumbie. Mémoire sociale et musicale dans les steelbands (Trinidad et Tobago)}

Nanterre, Société d'ethnologie, coll. « Hommes et musiques », 2012

Tommaso Montagnani

\section{RÉFÉRENCE}

Aurélie Helmlinger. Pan Jumbie. Mémoire sociale et musicale dans les steelbands (Trinidad et Tobago). Nanterre, Société d'ethnologie, coll. « Hommes et musiques », 2012. 


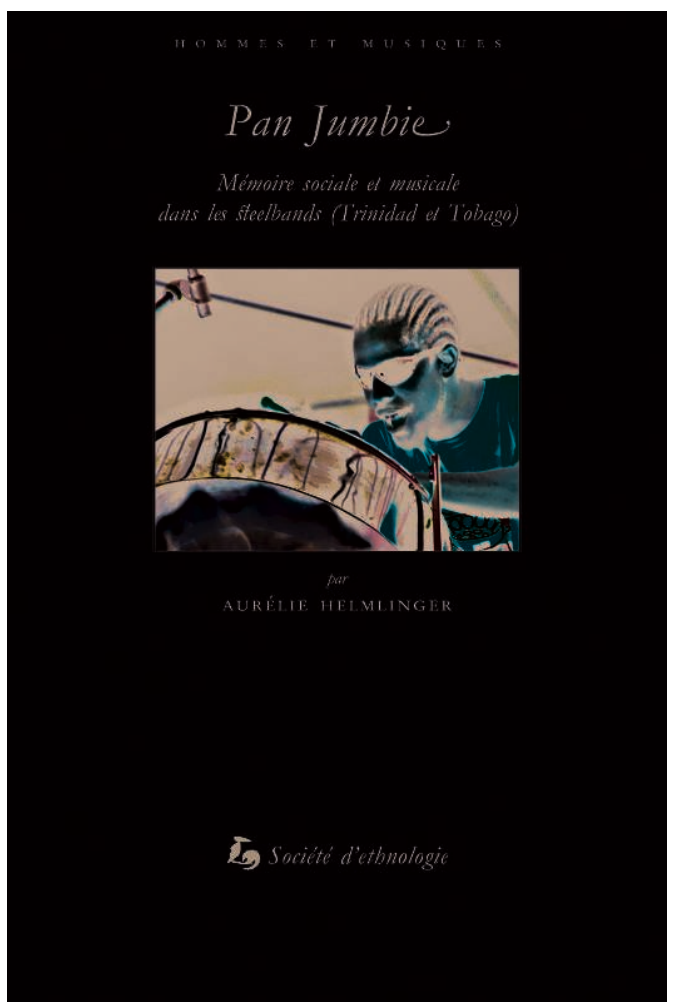

1 L'ethnomusicologue Aurélie Helmlinger est l'auteur d'un ouvrage sur la pratique du pan, instrument métallophone de Trinidad et Tobago issu de la récupération de bidons de pétrole. La musique de pan est exécutée par des orchestres appelés steelbands, actifs principalement pendant la période du carnaval.

2 Si l'ouvrage en question s'inscrit pleinement dans le courant de l'ethnomusicologie cognitive caractérisant une partie importante de la production scientifique actuelle de la discipline, d'autres aspects de la pratique du pan sont également pris en considération et analysés en profondeur par Helmlinger. Le texte approche la musique de pan de façon remarquablement exhaustive, ne délaissant à aucun moment les questions de société, de croyance et de pratique collective si importantes dans la vie des steelbands trinidadiens.

Dans la première partie du livre, l'auteur fournit une description de la composition sociale de Trinidad et Tobago tout au long de l'histoire du pays. Helmlinger présente la pratique musicale comme étant à la fois ciment de l'identité collective du pays et caractérisée par la diversité du complexe tissu social trinidadien. Dans le chapitre sur l'histoire et la société, elle inclut une importante référence au pan jumbie, l'esprit du pan, qui donne son titre à l'ouvrage. Les musiciens se disent souvent possédés ou dominés par cet esprit pendant leur performance, jusqu'à avoir la sensation de jouer sous l'influence d'un agent externe. Bien que les thématiques de croyance ne soient pas centrales dans le livre, Helmlinger analyse avec des outils extrêmement efficaces l'idée d'automatisme caractérisant une partie de l'apprentissage des séquences de mouvements nécessaires à l'exécution de la musique de pan.

4 La première partie du livre se poursuit par un chapitre sur l'organisation sociale des steelbands. Les hiérarchies internes à l'orchestre y sont analysées et décrites avec précision et clarté. Les facteurs pris en compte dans l'admission d'un musicien dans un orchestre sont expliqués dans toute leur complexité (des facteurs à la fois sociaux et 
purement musicaux), ainsi que les échelles de prestige, souvent fondées sur la difficulté des différentes typologies de pan. L'organologie des instruments peut en effet varier considérablement selon le registre et le rôle de chaque type de pan. Les questions d'esthétique musicale sont en outre développées dans le dernier chapitre de la première partie, où l'opposition entre musique locale et musique étrangère est décrite. Les steelbands sont extrêmement perméables aux musiques non autochtones, et les reprises de morceaux préexistants font partie de la pratique courante de ces ensembles. Le choix de l'accordage et de la sonorité des instruments (accordage traditionnel, avec une attaque précise ou moderne, caractérisé par un son plus riche en harmoniques et de longue durée) anime aussi les débats au sein des orchestres et représente souvent une prise de position nette et consciente en matière de choix esthétiques.

5 Dans la seconde partie du livre, intitulée "Mémorisation : données de terrain, approche cognitive ", l'auteur expose les résultats de ses études sur le fonctionnement de la mémoire musicale des steelbands. La capacité à appendre par cœur représente un enjeu fondamental pour les musiciens trinidadiens, et la valeur d'un instrumentiste de pan est mesurée non seulement à partir de sa technique et de son sens du rythme, mais aussi de la rapidité et de l'efficacité avec lesquelles il apprend de nouvelles parties.

6 La transmission repose sur ce que l'auteur définit comme une chaine de mémoire, un mécanisme pyramidal dans lequel on retrouve en partie les hiérarchies de l'orchestre : l'arrangeur, figure clé des steelbands, aussi important que le compositeur, transmet les parties à un premier cercle de musiciens. Ceux-ci sont alors chargés à leur tour de transmettre les parties à des groupes plus étendus d'instrumentistes.

7 En ce qui concerne la mémoire de chaque interprète, Helmlinger réussit à décrypter et à décrire avec finesse et précision le fonctionnement du processus de remémoration des parties à exécuter, et ceci grâce à une analyse rigoureuse de ses donnés ainsi qu'à une série d'expériences qu'elle a elle-même mises au point, inspirées de la psychologie cognitive. On apprend que l'ergonomie de l'instrument et la mémoire du mouvement des mailloches à l'intérieur du pan jouent un rôle fondamental, et que la mémoire visuelle et l'utilisation d'images sont aussi importantes que la mémoire musicale liée à la hauteur des sons. La mémoire des panistes repose sur une conception synesthésique dans laquelle deux sens, la vue et l'ouïe, participent à la recomposition mentale des gestes nécessaires à l'exécution des pièces. À sa capacité à localiser exactement la hauteur des sons à l'intérieur du bidon (capacité que seuls les panistes les plus expérimentés possèdent pleinement), l'interprète associe une appréhension visiospatiale reposant sur l'encodage des cartes mentales formées par les séquences de gestes à exécuter sur l'instrument. Non seulement donc, il existe une mémoire de l'emplacement des notes, mais aussi une mémoire des relations entre les emplacements. La mémoire individuelle est ensuite analysée par Helmlinger en rapport avec la pratique collective de l'orchestre : pendant la performance, les panistes jouent ensemble sous l'influence d'une sorte d'effet de mimétisme, où la proximité physique avec les autres membres de l'orchestre facilite le déclenchement de certains automatismes du geste musical.

8 Le livre est accompagné d'un CD-Rom contenant à la fois des vidéos (performances de steelbands) et d'autres supports multimédias (transcriptions, topologie des instruments, gammes et intervalles). Les animations multimédia du CD contribuent à l'efficacité et à la clarté des graphiques et des illustrations contenus dans le livre et à la 
compréhension des représentations visuelles des séquences de mouvements dans le pan.

9 Le travail d'Helmlinger avec les orchestres de pan de Trinidad et Tobago est novateur et très rigoureux en ce qui concerne les méthodologies d'analyse. Cet ouvrage offre une description détaillée de la pratique musicale des steelbands dont la portée dépasse le seul contexte des recherches de l'auteur: les concepts et les idées ici développés et utilisés constituent des outils précieux pour l'avancement du débat ethnomusicologique sur le fonctionnement de la mémoire musicale.

\section{AUTEURS}

\section{TOMMASO MONTAGNANI}

koboconcept@gmail.com 